\title{
'Ajde' and 'Hajde': Contexts of the Use of Balkanisms in Translations into South Slavic Languages
}

\author{
Kristýna DUFKOVÁ*
}

The South Slavic languages belong to the wider Slavic language family and as far as we know, the similarities among them are very extensive. On the other hand, there are many differences between the South Slavic languages and other Slavic branches (the East and the West Slavic languages). To mention just one of them, it is the influence of the Turkish or Ottoman Turkish language. The influence of the Turkish language is very extensive among the South Slavic languages, but not so among the East and West Slavic languages. Nevertheless, the influence of the Turkish language is not consistent even among particular South Slavic languages. The purpose of this paper is to present the differences between the South Slavic languages which are based on the different usage of exclamations 'ajde' and 'hajde' among particular South Slavic languages, as from our experience this seems to be significant. The aim of this paper is to explore the contexts of these two exclamations in the three South Slavic languages, to compare the contexts, and to highlight the different usage of these two words not just among particular South Slavic languages, but also within individual language systems. We have found that the dictionaries of the researched languages do not contain the differences we found out by our comparison. For precise use of these words in identical contexts, we decided to use a comparative analysis of interlingual translations. We have chosen three translations of the children's book Harry Potter and the Philosopher's Stone by J. K. Rowling, the Croatian, Serbian, and Macedonian ones. We have compared the contexts in which these words occur, and we found fundamental differences, whose explanation we offer in this article.

Keywords: South Slavic languages; Balkanism; haydi; children's literature; translation

\section{Introduction}

In this article we deal with Balkanisms 'ajde' and 'hajde' [xăjde] which occur as a lexeme with colloquial usage in many South Slavic languages. We focus on the context in which this word occurs according to the dictionaries, and we compare the usage found in dictionaries to the contexts in which these two words occur in translated children's literature. This topic is also interesting from a teacher's perspective. Among other subjects, at Masaryk University, Macedonian language and Balkan languages are also taught. Both also include translation from

\footnotetext{
* Assistant professor at the University of Hradec Králové; PhDc at Masaryk University, Brno. E-mail: kristyna.dufkova@seznam.cz; ORCID ID: https://orcid.org/0000-0002-0518-4254. (Received 17 October 2020; accepted 4 December 2020)
} 
some South Slavic language into Czech (and Slovak) and vice versa. According to our pedagogical experience, the translation of the word 'ajde' seems problematic. Aiming to outline the different contexts the word is used in, the study may help students improve their translations. Nevertheless, as we show below, the definitions and explanations in selected dictionaries of Croatian, Serbian, and Macedonian are limited. For our current work, we compare the original foreign language children's text, Harry Potter and the Philosopher's Stone by J. K. Rowling (2000), and its three translated versions into Croatian (Crnković 2014), Serbian (Roganović and Stamenković Roganović 2016), and Macedonian (Anonymous [2012?]), ${ }^{1}$ bearing in mind that the language of the children's book should be particularly authentic to evoke everyday speech as stated by Jenny Brumme $(2012,8)$. At the same time, the texts give us exactly the same context of the two selected words ('ajde' and 'hajde'), so we can consider the comparison as provable. Thus, we deal with the translation of the children's literature as a field of study. In other words, we first describe the need to analyze the language of the translated literature. Secondly, we analyze three of the translated texts. Finally, we discuss the meaning of the Balkanisms 'ajde' and 'hajde' and their usage in the three South Slavic translations of the selected children's book Harry Potter and the Philosopher's Stone, as well as highlighting the specificities, similarities, and differences of its usage.

\section{Methods}

The aim of this paper is to compare and contrast the usage of Balkanisms 'ajde' and 'hajde' in three languages-Macedonian, Serbian, and Croatian - and at the same time demonstrate the contexts in which the words occur in these languages. An integral part of this paper is also a description of the differences of contexts between individual languages. This paper uses the methods of contrastive linguistics. A framework of comparison is provided by an analysis of entire translation texts that have been translated into the South Slavic languages directly from English. It is therefore clear that using these translations, we are avoiding the possible effect of a negative transfer between related languages, as defined by Jan Čapek (2004) as a transfer of erroneous habits from the mother tongue or another foreign one into

\footnotetext{
${ }^{1}$ The translator of the Macedonian version is anonymous. This fan translation has been used in this paper because the official translation from the year 2002 is not considered to be of very high quality. It was last accessed on December 22, 2020 at this online forum: https:/forum.kajgana.com/threads/Хари-Потер.8283/page-64.
} 
another/different foreign language, based on Josef Hendrich et al. (1988) and Čestmír Liškar̆ (1970).

In the present paper, we also adhere to the principle of comparative linguistics. In order to realize the comparison in terms of comparative linguistics, the compared languages should be socio-culturally linked. The socio-cultural linking is more than obvious. It is well known that all three selected languages belong to the South Slavic language branch and at the same time all three were used in one common state (Yugoslavia). Moreover, Ottoman Turkish has a certain influence on all three selected languages. In our opinion, the contrastive approach to the language units is all-important for quality translations.

\section{Specificities of the Translation of Children's Literature}

For our comparison, we choose contemporary children's foreign literature because it is considered that when translating children's literature, clear and current language should be used, especially in direct speech (Brumme 2012, 8). It is generally agreed that translation of children's literature is a specific and relatively new area which has emerged from general translation studies and from comparative literature. It is necessary to mention that translations of children's literature have not received much attention in the past, unfortunately. This type of translation was not at the center of academic studies because it was thought that both the children's literature and the translations of the children's literature did not have as high a status as other types of literature and translations (O'Connoll 2006, 19). In recent decades, however, the situation has improved, and more attention has been paid to the child reader and the language in translation. The above-stated idea applies to minority language cultures and translations to these languages (O'Connoll 2006, 22). The methods of translation studies have been widely adopted in the field of translation of children's literature. However, there are some specificities that occur in the translation of this type of literature that should be mentioned. In addition to professional, linguistic, and cultural competence (Gavurova 2018, 73), when translating children's literature, it is necessary to consider the unique aspects of the child reader and his language. Zohar Shavit $(2006,25)$ points out that we should think about the translation of children's literature as a part of the transfer process, seeing that children's literature does not exist in a vacuum. Children's literature should be a part of the target literary system (just as the language used should be part of the target linguistic system). Judith Inggs (2003, 285) 
comments that whilst translating children's literature, "the dual role" is important, because of the "shaping [of] the child's cultural identity and world-view" and the "broadening [of] the child's knowledge and understanding of other cultures." Shavit (2006) points out that this type of literature is prone to adaptations and manipulations, and Reinbert Tabbert (2002) adds that due to readability, language purification and simplification appear in these types of translations. Cay Dollerup (2003, 99-100) mentions the importance of the fluency of the target language because children's literature is often read aloud. That point is also related to what Ronald Jobe (1996, 513) states about the role of "dual readership."

From many children's books, we have chosen Harry Potter and the Philosopher's Stone (Rowling 2000) and its translated versions (Crnković 2014; Roganović and Stamenković Roganović 2016; Anonymous [2012?]) because it corresponds to the issues we examine. The translations are relatively new, and the language used is contemporary. At the same time, the translation is not burdened by interference between the individual Slavic languages because the book was translated directly from English into all of them. Thus, it is possible to effectively prove how well this Balkanism is actually used in direct speech. For these reasons, we feel confident that translations of this text are suitable for our comparison.

From a cursory reading of these translations of the above-mentioned book, it is obvious that the translators encountered many difficulties and that in many cases they had to be very resourceful and linguistically inventive. Much has already been written about translation teasers in the book Harry Potter and the Philosopher's Stone (Jentsch 2001, 2002; Feral 2006). However, in the present paper, we do not focus on this attractive topic. In this paper, we look specifically at the usage of the words 'ajde' or 'hajde' and their contexts in the three translations, keeping in mind the specificities of the translation of the children's books.

As far as we know, no previous research has been done in this specific field of study.

\section{4. 'Ajde' and 'Hajde' as Balkanisms: Meaning}

From our perspective, there are three possible definitions of so-called Balkanism. In terms of the wider context, Balkanism can include a nonlinguistic meaning connected with the fragmentation of this geographical area (Todorova 2004) often in the minds associated with "a permanent state of cold or warm conflict between neighboring countries for territories with 
mixed populations" (Madgearu 2008, 3). It is related to Western stereotypes, which were produced by discourses in the context of the Former Yugoslav area (Hetemi 2015).

The second definition focuses on the linguistic point of view. Balkanism then is a language unit or a linguistic or cultural feature which is connected to the region of the Balkans (Friedman 2011, 275-289). In this regard, it is possible to mention phonological, morphosyntactic, and lexical Balkanisms. It is generally accepted that they were caused by common cultural and historical circumstances in the Middle Ages and early modern times (Gramelová 2014, 44-50). Among the Balkanisms, we can count, for example, the postpositive article, possessive dative, repetition of the object, lack of infinitive, or borrowed words from Latin, Greek, Slavic, or Turkish ${ }^{2}$ (Novák 1979; Kurzová 1995). The words selected for this study also fall into the latter category. ${ }^{3}$ At this point, what Victor A. Friedman stated (1994, 521) should be added, which goes as "[t]he Turkish lexicon in the various Balkan languages has undergone a variety of vicissitudes in terms of numbers, meanings and stylistic values."

And finally, we can think of Balkanism as a lexicon that is borrowed from so-called Balkan languages; for instance, some Balkanisms also penetrated the West Slavic languages, (e.g., bet’ár, dohán, kalauz, pagáč) (Hauptová 1970).

The words 'ajde' and 'hajde' are common Balkan hortative particles or interjections or verb forms for the imperative, depending on the specific use in the sentence. The standard translation in dictionaries is "come on, c'mon, let's. ..." This is evidenced by Eleni Bužarovska (2020) in the example "Ajde da se pitame na tova vol! — "Let's ask this ox!"

The lexeme 'ajde' with its variant 'hajde' is borrowed from Ottoman Turkish (hayde), هايدى (haydi), which is an univerbated sequence of the interjection هاى (hay), هیى (hey) 'to raise attention' and ده (de), دى (di, de) 'well then, forward, speak!' (Skok 1971, 649). The word is used in the Balkans, and of course among the South Slavic languages.

In Macedonian, forms ajde (ajde), ajdeme (ajdete) are used in colloquial language with the meaning of (1) command: ${ }^{4}$ e.g., “Ајде оди, не седи тука!” (Ajde odi, ne sedi tuka!) 'Come on, don’t sit here!’; “Ајде напиј се и на ништо не мисли!” (Ajde napij se i na ništo ne misli!) 'Come on, get drunk and don't think about anything!' We can use it, when (2) saying goodbye

\footnotetext{
${ }^{2}$ Words that are borrowed from Turkish are called Turkisms. The term is used, for example, by Schwarz and Blažek $(2011,40)$.

${ }^{3}$ For the wider discussion of terminology, see Stachowski 2019.

${ }^{4}$ The examples concerning the use of the said words are originals. Transliterations in the Latin script are given in the parentheses. The translations into English are marked by the single quotes and mine unless otherwise stated.
} 
and toasting: е.g., “Ајде со здравје!” (Ajde so zdravje!) ‘Вуе!’; “Ајде на здравје!” (Аjde na zdravje!) 'Cheers!' We can use it (3) when encouraging someone and for encouragement: e.g., “Ајде детенце!” (Ajde detence!) ‘Come on, baby!’; “Ајде мило!” (Ajde milo!) ‘Come on, honey!' It (4) expresses a wish: e.g., “Ајде да ми беа тука моите другари!” (Ajde da mi bea tuka moite drugari!) 'If only my friends were here!' It can be also used (5) in question sentences: e.g., “Ајде што е тоа од тебе?” (Ajde što e toa od tebe?) 'Come on. What is it about you?'5 Additionally, Christina E. Kramer and Liljana Mitkovska $(2011,109)$ note that the interjection 'ajde' is used with so-called da clause ${ }^{6}$ to form a type of imperative. The author gives these еxamples: “Ајде!” (Ajde!) ‘Come on, let’s ...'; “Ајде да пееме!” (Ajde da peeme!) ‘Come on, let's sing!'; “Ајде да одиме!” (Ajde da odime!) 'Come on, let's go!' The usage of the word 'ajde' is evidenced also by Kiril Koneski $(1999,22)$.

In Serbian, the particle 'ajde' or 'hajde' can apply to all persons, and it can be used to pronounce various imperative meanings, for instance an encouragement or command to tell something (Bogdanović 1987, 256). This form can have the meaning of imperative of the verb to go, or it is a simple imperative word for urging, to encourage any action (Stevanovic 1970, 706). An imperative can be expressed by the periphrastic construction hajde + present form: e.g., “Хајде да певамо.” (Најde da pevamo.) or “Хајде да запевамо.” (Hajde da zapevamo.) vs. “Певајмо!” (Рevajmo!) or “Запевајмо!” (Zapevajmo!), all of them with the meaning 'Let's sing' (Piper and Ivič 2005, 663).

In Croatian, the following forms can be used: hàjde, hàjdemo, hàjdmo, àjmo, hàjmo, àjde to (1) encourage someone, with the meaning 'Come on, go, let's go!' This form can (2) also emphasize the imperative of the verb of movement, e.g., "Hajde, požuri!" 'Come on, hurry up!' Very impressive are the reduplicated forms hajdedë or ajdedë with the meaning of indifferent and uninterested satisfaction or agreeing with what the speaker is saying. For instance, “A: Nisam miran dok ne postanem najbolji šahist u zemlji. B: Hajdede probaj." "A: I will not be calm until I become the best chess player in the country. B: Let's try it. ${ }^{8}$ More detailed definition of "de" is offered by Petar Skok $(1971,386)$.

\footnotetext{
${ }^{5}$ DRMJ (Digitalen rečnik na makedonskiot jazik), s.vv. "ајде," “ајдете,” accessed December 22, 2020, http://www.makedonski.info.

${ }^{6}$ The da clause or да-construction uses the following sequence: conjugated verb + да + conjugated verb (Kramer and Mitkovska 2011, 75-76).

${ }^{7}$ The mark above the letter à indicates the short rising accent in Croatian.

${ }^{8}$ HJP (Hrvatski jezični portal), s.v. “hajdede," accessed December 22, 2020, http://hjp.znanje.hr.
} 


\section{Research Questions and Hypothesis}

Based on our pedagogical experience on the subjects of Macedonian language and Balkan languages which are taught at Masaryk University in Brno and in which translation is also discussed and taught, the usage and the translation of the afore-mentioned words seems to be problematic for students, especially when such expression does not occur in their mother tongue. Thus, the important question to study seems to be: Does the width of the field of meaning of the words 'ajde' and 'hajde' differ on the one hand in dictionaries and on the other hand in written translated texts? And extending the question further, how much does the use of the words differ in the examined texts? The answer to the latter shows us to what extent the usage among several South Slavic languages (in this specific aspect) is different.

In order to address this question properly, we first gathered data from four texts and listed them in Table 1. Then we specified the common usage of the word in each of the texts, and we pointed out problematic phenomena. After that, we offered a description of newly identified contexts of the word in each of the texts that we focused on.

In our opinion, it should be mentioned that in some regions of the Balkans, the Turkish influence has been stronger than in others, especially in urban or rural areas with rather heavy Turkish colonization (Kahl 2014, 314). Thus, the following hypothesis can be formulated: the texts written in the language that was under a wider influence of Turkish or Ottoman Turkish will contain a greater number of occurrences of the exanimated word and wider context in which this word occurs than the texts written in a less influenced language.

\section{Data and Findings}

For this study, we analyzed the data collected from individual translated texts (Crnković 2014; Roganović and Stamenković Roganović 2016; Anonymous [2012?]), as well as from an original of the book Harry Potter and the Philosopher's Stone (Rowling 2000).

Table 1 includes the numbers of the chapters, which the excerpts are taken from, in the first column; the original excerpts from Harry Potter and the Philosopher's Stone in English in the second column; the excerpts from Хари Потер и Каменот на мудроста (Hari Poter i Kamenot na mudrosta) in Macedonian in the third column; the excerpts from Хари Потер $u$ Камен мудрости (Hari Poter i Kamen mudrosti) in Serbian in the fourth column; and the excerpts from Harry Potter i Kamen mudraca in Croatian in the fifth column. 
It should be noted that unlike the other columns, the text in the second column is always filled in because it is the original text. In the next three columns, we list only the sentences in which the search word occurred in the given language. Therefore, some spaces in this table are empty.

The last row of table 1 shows the sum of the contexts in which the researched term appeared in the individual languages. The characteristics of the underlined words are explained, and the data are presented in table 1.

Table 1. Found data and contexts in translations ${ }^{9}$

\begin{tabular}{|c|c|c|c|c|}
\hline Chapter & $\begin{array}{l}\text { English original } \\
\text { (Rowling 2000) }\end{array}$ & $\begin{array}{l}\text { Macedonian } \\
\text { translation } \\
\text { (Anonymous } \\
{[2012 ?] \text { ) }}\end{array}$ & $\begin{array}{l}\text { Serbian } \\
\text { translation } \\
\text { (Roganović and } \\
\text { Stamenković } \\
\text { Roganović 2016) }\end{array}$ & $\begin{array}{l}\text { Croatian } \\
\text { translation } \\
\text { (Crnković 2014) }\end{array}$ \\
\hline 2 & $\begin{array}{l}\text { Well - give him } \\
\text { here, Hagrid - } \\
\text { we'd better get } \\
\text { this over with. }\end{array}$ & $\begin{array}{l}\text { Добро... стави го } \\
\text { тука, Хагрид... } \\
\text { ајде што побрзо } \\
\text { да го завршиме } \\
\text { ова. }\end{array}$ & & \\
\hline 2 & Up! Get up! Now! & $\begin{array}{l}\text { Ајде! Станувај! } \\
\text { Сега! }\end{array}$ & $\begin{array}{l}\text { Hajde! Ustaj! } \\
\text { Smesta! }\end{array}$ & \\
\hline 2 & $\begin{array}{l}\text { Well, get a move } \\
\text { on, I want you to } \\
\text { look after the } \\
\text { bacon. }\end{array}$ & $\begin{array}{l}\text { Ајде, мрдни, } \\
\text { сакам да ја } \\
\text { припазиш } \\
\text { сланината. }\end{array}$ & $\begin{array}{l}\text { Hajde, mrdni, } \\
\text { hoću da pripaziš } \\
\text { na slaninu. }\end{array}$ & $\begin{array}{l}\text { Hajde, požuri se } \\
\text { da pripaziš na } \\
\text { slaninu u tavi. }\end{array}$ \\
\hline 3 & $\begin{array}{l}\text { Come on! } \\
\text { Everyone out! }\end{array}$ & $\begin{array}{l}\text { Ајде! Сите } \\
\text { надвор! }\end{array}$ & $\begin{array}{l}\text { Hajde! Izlazite } \\
\text { svi! }\end{array}$ & $\begin{array}{l}\text { Hajde, svi van iz } \\
\text { kola! }\end{array}$ \\
\hline 3 & $\begin{array}{l}\text { "I've already got } \\
\text { us some rations," } \\
\text { said Uncle } \\
\text { Vernon, "so all } \\
\text { aboard!" }\end{array}$ & $\begin{array}{l}\text { “Веќе зедов } \\
\text { резерви храна за } \\
\text { нас,” рече тетин } \\
\text { Вернон, “така } \\
\text { што ајде, сите } \\
\text { влегувајте!” }\end{array}$ & & \\
\hline 5 & $\begin{array}{l}\text { Got everythin'? } \\
\text { Come on, then. }\end{array}$ & $\begin{array}{l}\text { Зема све? Ајде } \\
\text { тоаш. }\end{array}$ & $\begin{array}{l}\text { 'Si sve uz'o? } \\
\text { Ajdemo, onda. }\end{array}$ & $\begin{array}{l}\text { Je } 1 \text { ti sad sve } \\
\text { jasno? Ajde, } \\
\text { idemo! }\end{array}$ \\
\hline 5 & $\begin{array}{l}\text { Must get on - lots } \\
\text { ter buy. Come on, } \\
\text { Harry. }\end{array}$ & $\begin{array}{l}\text { Мора да одиме... } \\
\text { има многу да } \\
\text { купиме. Ајде, } \\
\text { Хари. }\end{array}$ & $\begin{array}{l}\text { Mora' d' idemo - } \\
\text { još puno ima da } \\
\text { pazarimo. Hajde, } \\
\text { Hari. }\end{array}$ & \\
\hline
\end{tabular}

\footnotetext{
${ }^{9}$ Some of the words in table 1 have been underlined by the author of this paper. Their use is specific as explained
} below. 


\begin{tabular}{|c|c|c|c|c|}
\hline 5 & $\begin{array}{l}\text { Come on, back in } \\
\text { this infernal cart. }\end{array}$ & & $\begin{array}{l}\text { Ajde, idemo } \\
\text { natrag u taj } \\
\text { pakleni vagon. }\end{array}$ & $\begin{array}{l}\text { Ajdemo sad opet } \\
\text { na ta prokleta } \\
\text { kolica. }\end{array}$ \\
\hline 5 & $\begin{array}{l}\text { Well, now - Mr } \\
\text { Potter. Let me } \\
\text { see. }\end{array}$ & $\begin{array}{l}\text { Па, ајде, } \\
\text { господине } \\
\text { Потер. Дај да } \\
\text { видам. }\end{array}$ & & \\
\hline 5 & $\begin{array}{l}\text { Right then, } \mathrm{Mr} \\
\text { Potter. Try this } \\
\text { one. }\end{array}$ & $\begin{array}{l}\text { Ајде сега, } \\
\text { господине } \\
\text { Потер. Пробај го } \\
\text { ова. }\end{array}$ & & \\
\hline 5 & $\begin{array}{l}\text { Go on, go on, try } \\
\text { it out. }\end{array}$ & $\begin{array}{l}\text { Ајде, ајде, пробај } \\
\text { го. }\end{array}$ & $\begin{array}{l}\text { Hajde, hajde, } \\
\text { probajte ga. }\end{array}$ & $\begin{array}{l}\text { Hajde, hajde, } \\
\text { isprobajte ga! }\end{array}$ \\
\hline 6 & $\begin{array}{l}\text { All right, Percy, } \\
\text { you go first. }\end{array}$ & $\begin{array}{l}\text { Ајде, Перси, оди } \\
\text { прв. }\end{array}$ & & \\
\hline 6 & $\begin{array}{l}\text { Go on, go now } \\
\text { before Ron. }\end{array}$ & $\begin{array}{l}\text { Оди ајде, оди } \\
\text { сега пред Рон. }\end{array}$ & $\begin{array}{l}\text { Hajde sada, pođi } \\
\text { ispred Rona. }\end{array}$ & \\
\hline 6 & $\begin{array}{l}\text { Give us a look, } \\
\text { Lee, go on. }\end{array}$ & $\begin{array}{l}\text { Дај да sирнеме, } \\
\text { Ли, ајде. }\end{array}$ & $\begin{array}{l}\text { Daj nam da } \\
\text { vidimo, } \mathrm{Li} \text {, hajde. }\end{array}$ & \\
\hline 6 & Go on - & Ајде. & Hajde, uzmi... & \\
\hline 6 & $\begin{array}{l}\text { Go on, have a } \\
\text { pasty. }\end{array}$ & Ајде, земи пита. & $\begin{array}{l}\text { Hajde bre, uzmi } \\
\text { pitu. }\end{array}$ & \\
\hline 6 & Let's see it, then. & Ајде да видиме. & & $\begin{array}{l}\text { Hajde baš da } \\
\text { vidimo! }\end{array}$ \\
\hline 7 & $\begin{array}{l}\text { Forgive and } \\
\text { forget. }\end{array}$ & & $\begin{array}{l}\text { Hajde da } \\
\text { oprostimo i } \\
\text { zaboravimo. }\end{array}$ & \\
\hline 7 & Move along now. & $\begin{array}{l}\text { Ајде сега, движе } \\
\text { те се. }\end{array}$ & Hajde, mičite se. & $\begin{array}{l}\text { Hajde radije } \\
\text { odlazite! }\end{array}$ \\
\hline 7 & Now, form a line. & & $\begin{array}{l}\text { Hajde, postrojite } \\
\text { se. }\end{array}$ & \\
\hline 7 & $\begin{array}{l}\text { And now, before } \\
\text { we go to bed, let } \\
\text { us sing the school } \\
\text { song! }\end{array}$ & $\begin{array}{l}\text { А сега, пред да } \\
\text { одиме во кревет, } \\
\text { ајде да ја } \\
\text { испееме } \\
\text { училишната } \\
\text { песна! }\end{array}$ & & \\
\hline 7 & $\begin{array}{l}\text { And now, } \\
\text { bedtime. Off you } \\
\text { trot! }\end{array}$ & $\begin{array}{l}\text { А сега е време за } \\
\text { кревет. Ајде, } \\
\text { трк! }\end{array}$ & & \\
\hline 8 & $\begin{array}{l}\text { Let's try again. } \\
\text { Potter, where } \\
\text { would you look if } \\
\text { I told you to find } \\
\text { me bezoar? }\end{array}$ & $\begin{array}{l}\text { Ајде да се } \\
\text { обидеме пак. } \\
\text { Потер, каде би } \\
\text { барал ти кога јас } \\
\text { би ти рекол да } \\
\text { ми најдеш } \\
\text { безоар? }\end{array}$ & & $\begin{array}{l}\text { Hajde, Potter, da } \\
\text { još jednom } \\
\text { pokušamo. Na } \\
\text { koju ćeš stranu } \\
\text { pogledati ako ti } \\
\text { kažem da potražiš } \\
\text { bezoar? }\end{array}$ \\
\hline
\end{tabular}




\begin{tabular}{|c|c|c|c|c|}
\hline 9 & $\begin{array}{l}\text { Come on, hurry } \\
\text { up. }\end{array}$ & Ајде, побрзајте. & Hajde, požurite. & \\
\hline 9 & $\begin{array}{l}\text { Come on, boy - } \\
\text { it's all right, up } \\
\text { you get. }\end{array}$ & $\begin{array}{l}\text { Ајде, момче... } \\
\text { добро е, } \\
\text { станувај. }\end{array}$ & $\begin{array}{l}\text { Hajde, dečače, sve } \\
\text { je u redu, ustani. }\end{array}$ & $\begin{array}{l}\text { Hajde, dečko... } \\
\text { sve je u redu, } \\
\text { ustaj! }\end{array}$ \\
\hline 9 & Come on, dear. & Ајде, душо. & Hajde, dušice. & $\begin{array}{l}\text { Hajdemo, } \\
\text { dragoviću! }\end{array}$ \\
\hline 9 & $\begin{array}{l}\text { Come and get it, } \\
\text { Potter! }\end{array}$ & & $\begin{array}{l}\text { Hajde, Poteru, } \\
\text { dođi i uzmi je! }\end{array}$ & \\
\hline 9 & We'd better go. & Ајде да одиме. & & \\
\hline 9 & $\begin{array}{l}\text { "Come on," he } \\
\text { said to Ron. }\end{array}$ & $\begin{array}{l}\text { “Ајде,” му рече } \\
\text { тој на Рон. }\end{array}$ & $\begin{array}{l}\text { Hajde - reče } \\
\text { Ronu. }\end{array}$ & \\
\hline 9 & Let's go. & Ајде, одиме. & Hajdemo. & \\
\hline 10 & $\begin{array}{l}\text { Let's try you out } \\
\text { with a few of } \\
\text { these. }\end{array}$ & $\begin{array}{l}\text { Ајде да те } \\
\text { видиме со } \\
\text { неколку од овие. }\end{array}$ & & $\begin{array}{l}\text { Hajde da se radije } \\
\text { ogledaš s ovima. }\end{array}$ \\
\hline 10 & $\begin{array}{l}\text { You do it, then, if } \\
\text { you're so clever. }\end{array}$ & $\begin{array}{l}\text { Ајде ти кажи, } \\
\text { кога си толку } \\
\text { паметна. }\end{array}$ & & \\
\hline 10 & $\begin{array}{l}\text { Stay close behind } \\
\text { me, now! }\end{array}$ & $\begin{array}{l}\text { Ајде, застанете } \\
\text { веднаш зад мене! }\end{array}$ & & \\
\hline 10 & $\begin{array}{l}\text { "Oh, all right," he } \\
\text { snapped. }\end{array}$ & $\begin{array}{l}\text { “Добро, ајде,” } \\
\text { одврати грубо. }\end{array}$ & & \\
\hline 10 & $\begin{array}{l}\text { Come on, run, } \\
\text { run! }\end{array}$ & $\begin{array}{l}\text { Ајде, трчај, } \\
\text { трчај! }\end{array}$ & Hajde, trči, trči! & $\begin{array}{l}\text { Hajde, bježi, } \\
\text { bježi! }\end{array}$ \\
\hline 11 & $\begin{array}{l}\text { Honestly, } \\
\text { Hermione, you } \\
\text { think all teachers } \\
\text { are saints or } \\
\text { something }[\ldots]\end{array}$ & & $\begin{array}{l}\text { Ma hajde, } \\
\text { Hermiona, da li ti } \\
\text { stvarno misliš da } \\
\text { su svi profesori } \\
\text { sveci ili nešto } \\
\text { slično? }\end{array}$ & \\
\hline 11 & $\begin{array}{l}\text { come on, now, } \\
\text { Angelina }\end{array}$ & ајде, Анџелина... & $\begin{array}{l}\text { hajde, sad, } \\
\text { Anđelina... }\end{array}$ & $\begin{array}{l}\text { hajde samo, } \\
\text { Angelina... }\end{array}$ \\
\hline 11 & $\begin{array}{l}\text { Budge up there, } \\
\text { move along. }\end{array}$ & & $\begin{array}{l}\text { Hajde de, miči se, } \\
\text { pomer' se već } \\
\text { jednom! }\end{array}$ & \\
\hline 11 & $\begin{array}{l}\text { Come on, } \\
\text { Hermione. }\end{array}$ & & Hajde, Hermiona. & $\begin{array}{l}\text { Hajde, hajde, } \\
\text { Hermiona! }\end{array}$ \\
\hline 12 & $\begin{array}{l}\text { Move along, all of } \\
\text { you! }\end{array}$ & $\begin{array}{l}\text { Ајде сега сите } \\
\text { одете. }\end{array}$ & & $\begin{array}{l}\text { Hajde sad, } \\
\text { prolazite svi dalje! }\end{array}$ \\
\hline 12 & $\begin{array}{l}\text { Come on, cheer } \\
\text { up, it's nearly } \\
\text { Christmas. }\end{array}$ & $\begin{array}{l}\text { Ај разведрете се, } \\
\text { иде Божиќ. }\end{array}$ & $\begin{array}{l}\text { Ma ajde, razvedri } \\
\text { se, već je skoro } \\
\text { Božić. }\end{array}$ & $\begin{array}{l}\text { Ajde, veselite se } \\
\text { rade, Božić je } \\
\text { pred vratima. }\end{array}$ \\
\hline 12 & $\begin{array}{l}\text { You'd better get } \\
\text { out, then. Go on - } \\
\text { out! }\end{array}$ & $\begin{array}{l}\text { Тогаш подобро } \\
\text { излези од тука. } \\
\text { Ајде - надвор! }\end{array}$ & $\begin{array}{l}\text { Onda izađi. Hajde } \\
\text { - briši! }\end{array}$ & $\begin{array}{l}\text { Onda odlazi } \\
\text { odavde! Hajde... } \\
\text { briši! }\end{array}$ \\
\hline
\end{tabular}




\begin{tabular}{|c|c|c|c|c|}
\hline 12 & $\begin{array}{l}\text { Come on, get it } \\
\text { on, they're lovely } \\
\text { and warm. }\end{array}$ & $\begin{array}{l}\text { Ајде, облечи го, } \\
\text { толку се } \\
\text { прекрасни и } \\
\text { топли. }\end{array}$ & $\begin{array}{l}\text { Hajde, obuci ga, } \\
\text { baš su lepi i topli. }\end{array}$ & $\begin{array}{l}\text { Hajde, obuci ga, u } \\
\text { njima je zbilja } \\
\text { lijepo i toplo. }\end{array}$ \\
\hline 12 & $\begin{array}{l}\text { Get it on, Percy, } \\
\text { come on, we're all } \\
\text { wearing ours. }\end{array}$ & $\begin{array}{l}\text { Стави го, Перси, } \\
\text { ајде, сите ние ги } \\
\text { носиме нашите. }\end{array}$ & $\begin{array}{l}\text { Obuci ga, Persi, } \\
\text { hajde, svi mi već } \\
\text { nosimo svoje. }\end{array}$ & $\begin{array}{l}\text { Hajde, Percy, } \\
\text { obuci ga, svi smo } \\
\text { mi obukli svoje. }\end{array}$ \\
\hline 12 & $\begin{array}{l}\text { Let's forget it and } \\
\text { go back. }\end{array}$ & $\begin{array}{l}\text { Ај да баталиме и } \\
\text { да се вратиме } \\
\text { назад. }\end{array}$ & $\begin{array}{l}\text { Hajde da batalimo } \\
\text { sve i vratimo se } \\
\text { nazad. }\end{array}$ & \\
\hline 12 & $\begin{array}{l}\text { Look in it } \\
\text { properly, go on, } \\
\text { stand where I am. }\end{array}$ & $\begin{array}{l}\text { Погледни убаво, } \\
\text { ајде, застани на } \\
\text { моето место. }\end{array}$ & & \\
\hline 12 & Come on. & Ајде. & Hajdemo. & Hajdemo! \\
\hline 13 & Come on, Harry! & Ајде, Хари! & Hajde, Hari! & \\
\hline 13 & $\begin{array}{l}\text { Let's find an } \\
\text { empty room. }\end{array}$ & $\begin{array}{l}\text { Ајде да најдеме } \\
\text { празна соба. }\end{array}$ & $\begin{array}{l}\text { Hajdemo u neku } \\
\text { praznu sobu. }\end{array}$ & $\begin{array}{l}\text { Hajdemo u neku } \\
\text { praznu sobu. }\end{array}$ \\
\hline 14 & $\begin{array}{l}\text { Oh, come on, } \\
\text { Hagrid, you might } \\
\text { not want to tell us. }\end{array}$ & $\begin{array}{l}\text { Ма ајде, Хагрид, } \\
\text { можеби не сакаш } \\
\text { да ни кажеш. }\end{array}$ & & \\
\hline 14 & $\begin{array}{l}\text { Come on - I shall } \\
\text { see Professor } \\
\text { Snape about you, } \\
\text { Malfoy! }\end{array}$ & $\begin{array}{l}\text { Ајде... ќе му } \\
\text { кажам на } \\
\text { професорот } \\
\text { Снејп за тебе, } \\
\text { Малфој! }\end{array}$ & $\begin{array}{l}\text { Hajde... moraću } \\
\text { da popričam s } \\
\text { profesorom } \\
\text { Snejpom o tebi, } \\
\text { Melfoje! }\end{array}$ & $\begin{array}{l}\text { Hajdemo... još ću } \\
\text { ja i s profesorom } \\
\text { Snapeom } \\
\text { porazgovarati o } \\
\text { vama, Malfoye! }\end{array}$ \\
\hline 15 & $\begin{array}{l}\text { Now get back to } \\
\text { bed, all of you. }\end{array}$ & $\begin{array}{l}\text { Ајде сега сите } \\
\text { назад во кревет. }\end{array}$ & & \\
\hline 15 & Go on! & Ајде. & Ajde! & Ajde! \\
\hline 15 & $\begin{array}{l}\text { So, be careful - } \\
\text { let's go! }\end{array}$ & $\begin{array}{l}\text { И пазете се... aj, } \\
\text { одиме. }\end{array}$ & $\begin{array}{l}\text { Dakle, budite } \\
\text { pažljivi... ajde, } \\
\text { idemo. }\end{array}$ & $\begin{array}{l}\text { Dobro, ajdete sad } \\
\text { za mnom, al } \\
\text { oprezno! }\end{array}$ \\
\hline 16 & $\begin{array}{l}\text { Oh, come off it, } \\
\text { you don't think } \\
\text { we'd let you go } \\
\text { alone? }\end{array}$ & $\begin{array}{l}\text { Ма ајде, Хари, } \\
\text { мислиш дека би } \\
\text { те оставиле да } \\
\text { одиш сам? }\end{array}$ & & \\
\hline 16 & $\begin{array}{l}\text { Go on then, try } \\
\text { and hit me! }\end{array}$ & $\begin{array}{l}\text { Ајде тогаш, } \\
\text { пробај удри ме! }\end{array}$ & $\begin{array}{l}\text { Hajde, onda, } \\
\text { probajte da me } \\
\text { udarite! }\end{array}$ & $\begin{array}{l}\text { Pa dobro, hajde, } \\
\text { udari me! }\end{array}$ \\
\hline 16 & $\begin{array}{l}\text { Oh, let's kick her, } \\
\text { just this once. }\end{array}$ & $\begin{array}{l}\text { Ајде да ја } \\
\text { клоцнеме, само } \\
\text { овој пат. }\end{array}$ & $\begin{array}{l}\text { Hajde da je } \\
\text { šutnemo, bar } \\
\text { jednom. }\end{array}$ & $\begin{array}{l}\text { Uh, hajde da je } \\
\text { bar ovaj put } \\
\text { udarimo nogom. }\end{array}$ \\
\hline 16 & $\begin{array}{l}\text { Come on, } \\
\text { Hermione! }\end{array}$ & Ајде, Хермајни! & Hajde, Hermiona! & $\begin{array}{l}\text { Hajde, skači i ti, } \\
\text { Hermiona! }\end{array}$ \\
\hline 16 & $\begin{array}{l}\text { Leaves you free to } \\
\text { take the bishop, } \\
\text { Hermione, go on. }\end{array}$ & $\begin{array}{l}\text { Сега можеш да } \\
\text { им го земеш оној } \\
\text { ловец, Хермајни, } \\
\text { ајде оди. }\end{array}$ & $\begin{array}{l}\text { Sada imaš } \\
\text { slobodan prostor } \\
\text { da uzmeš tog }\end{array}$ & $\begin{array}{l}\text { Ali sad ti, } \\
\text { Hermiona, možeš } \\
\text { slobodno uzeti }\end{array}$ \\
\hline
\end{tabular}




\begin{tabular}{|c|c|c|c|c|}
\hline & & & $\begin{array}{l}\text { lovca; hajde, } \\
\text { Hermiona. }\end{array}$ & $\begin{array}{l}\text { onog lovea, hajde, } \\
\text { uzmi ga! }\end{array}$ \\
\hline 16 & Go on. & Ајде, оди. & & \\
\hline 16 & $\begin{array}{l}\text { Come on, I can't } \\
\text { breathe. }\end{array}$ & $\begin{array}{l}\text { Ајде, не можам } \\
\text { да дишам. }\end{array}$ & $\begin{array}{l}\text { Hajdemo, ne } \\
\text { mogu da dišem. }\end{array}$ & \\
\hline 17 & $\begin{array}{l}\text { "Oh, very well," } \\
\text { she said. }\end{array}$ & $\begin{array}{l}\text { “О, добро, ајде,” } \\
\text { рече таа. }\end{array}$ & & \\
\hline 17 & $\begin{array}{l}\text { Have a Chocolate } \\
\text { Frog, I've got } \\
\text { loads... }\end{array}$ & & $\begin{array}{l}\text { Hajde, uzmi } \\
\text { čokoladnu žabicu, } \\
\text { imam ih na } \\
\text { tone... }\end{array}$ & \\
\hline 17 & $\begin{array}{l}\text { Hurry up, boy, we } \\
\text { haven't got all } \\
\text { day. }\end{array}$ & & & $\begin{array}{l}\text { Hajde požuri, } \\
\text { dečko, nemamo } \\
\text { cijeli dan } \\
\text { vremena. }\end{array}$ \\
\hline & 64 & $55(52+3)$ & $42(37+5)$ & $29(24+5)$ \\
\hline
\end{tabular}

A closer look at the data indicates that the majority of the occurrences were discovered in the Macedonian text, fewer in the Serbian text, and the fewest in the Croatian text. So, table 1 demonstrates that the use of the word 'ajde' is the most common in the Macedonian text; therefore, we can conclude that we find the broadest contexts of the usage of 'ajde' in Macedonian. Along with the contexts and examples which appear in Macedonian textbooks and dictionaries, the following points can also be added based on table 1:

(1) the usage of 'ajde' as an incentive and encouragement, e.g., “Ајде, оди.” (Ajde, odi.) "Go on.”; or an interjection of incentives, e.g., "Ма ајде.” (Ма ajde.) "Oh, come off it";

(2) the expression of the imperative of the verb of movement, e.g., “Ајде сега сите назад во кревет." (Ajde sega site nazad vo krevet.) "Now get back to bed, all of you.”; "Ајде сега сите одете.” (Ajde sega site odete.) “Move along, all of you!”; “Ајде! Станувај! Сега!” (Ajde! Stanuvaj! Sega!) “Up! Get up! Now!”; “А сега е време за кревет. Ајде, трк.” (A sega e vreme za krevet. Ajde, trk.) “And now, bedtime. Off you trot!”; “Ајде, застанете веднаш зад мене.” (Ajde zastanete vednaš zad mene.) "Stay close behind me, now.”;

(3) the incentive not expressed by a verb in the original text, e.g., “"Веќе зедов резерви храна за нас,' рече тетин Вернон, 'така што ајде, сите влегувајте.”' (“Vekje zedov rezervi hrana za nas," reče tetin Vernon. "taka što ajde, site vleguvajte.”) “'I've already got us some rations,' said Uncle Vernon, 'so all aboard."”;

(4) expression of emotions and attitudes of the speaker, interjections and adverbs, for instance 'well' and 'right,' e.g., “Па, ајде, господине Потер. Дај да видам.” (Ра, ajde, 
gospodine Poter. Daj da vidam.) “Well, now - Mr Potter. Let me see.”; “Ајде, мрдни, сакам да ја припазиш сланината.” (Ajde, mrdni, sakam da ja pripaziš slaninata.) “Well, get a move on, I want you to look after the bacon.”; “Ајде сега, господине Потер. Пробај го ова.” (Ајdе sega, gospodine Poter. Probaj go ova.) "Right then, Mr Potter. Try this one.”; “Ајде, Перси, оди прв.” (Ajde, Persi, odi prv.) “All right, Percy, you go first.”; ““Добро, ајде,’ одврати грубо.” (“Dobro, ajde,” odvrati grubo.) ““Oh, all right,' he snapped.”;

(5) the da-construction as an expression of the conditional tense, e.g., “Добро... стави го тука, Хагрид... ајде што побрзо да го завршиме ова.” (Dobro... stavi go tuka, Hagrid... ajde što pobrzo da go završime ova.) "Well - give him here, Hagrid - we'd better get this over with.”; “Ајде да одиме.” (Ajde da odime.) "We'd better go.”

Thus, in addition to translations that usually appear in dictionaries (come on, c'mon, let's), we could add frequent phrases for translation which resulted from this research: go on, move, get, up, off, etc. Furthermore, our results demonstrated that it is possible to use the word 'ajde' when using imperative. In such constructions, the word 'ajde' may not only replace the verb in the imperative form (as shown, e.g., in this sentence: “Ајде сега сите назад во кревет." (Ajde sega site nazad vo krevet.) "Now get back to bed, all of you."). According to our research, we can also translate the imperative expression by the word 'ajde' plus a full-meaning verb in the imperative, for example 'ajde' + 'odi' (ajde + go) instead of one verb 'go on': “Сега можеш да им го земеш оној ловец, Хермајни, ајде оди.” (Sega možeš da im go zemeš onoj lovec, Hermajni, ajde odi.) "Leaves you free to take the bishop, Hermione, go on." Our research suggests that the use of the word 'ajde' in Macedonian may be a symptom of a sarcastic statement of 'do it yourself to get it done,' e.g., “Ајде ти кажи, кога си толку паметна.” (Ajde ti kaži, koga si tolku pametna.) "You do it then, if you're so clever."

While studying the Macedonian text, we also came across the expression ' $a j$,' which replaces the word 'ajde' in certain contexts. This particle is used "to emphasize the following information that expresses different emotional relationships: delight, wonder, sadness, etc."10 This particle occurred in the examined text twice in Hagrid's speech. Hagrid is a character who does not use standard language either in the original text-he uses a variety of language, a sociolect. An example is “Ај разведрете се, иде Божиќ.” (Aj razvedrete se, ide Božik'.) “Come on, cheer up, it's nearly Christmas." The third occurrence of this specific word can be found in

\footnotetext{
${ }^{10}$ DRMJ (Digitalen rečnik na makedonskiot jazik), s.v. “aj,” accessed December 12, 2020, http://drmj.eu.
} 
a speech by Ron, one of the students, who is eleven years old and who does not use standard language, either, e.g., “Ај да баталиме и да се вратиме назад.” (Аj da batalime i da se vratime nazad.) "Let's forget it and go back."

The total number of occurrences of 'ajde' or its variant is 55 in the Macedonian text, with the word ' $a j$ ' used three times and the word form 'ajde' used 52 times. Additionally, in the Macedonian text, it is also remarkable that it is not necessary to translate all the expressions come on, c'mon, let's as 'ajde' or as the constructions with 'ajde,' e.g., "Па, ајде, господине Потер. Дај да видам.” (Рa, ajde, gospodine Poter. Daj da vidam.) “Well, now - Mr Potter. Let me see." In this article, we only draw attention to this fact, but we do not deal with it more in depth.

The second text that was investigated was the Serbian translation of the English original. In Serbian, there are two similar particles: 'ajde' and 'hajde.' Both can be applied to any person. As far as we know, these particles can be used to convey various imperative meanings. In our research, we listed all contexts that were found in the text. In addition to the translations of the expressions come on, c'mon, and let's which were found, other constructions should be mentioned too. The explanation of the usage is as follows:

(1) the expression of the imperative of the verb of movement, e.g., "Hajde! Ustaj! Smesta!” "Up! Get up! Now!”; "Hajde, mičite se." "Move along now.”; “Hajde, Poteru, dođi i uzmi je!" "Come and get it, Potter!”; "Hajde de, miči se, pomer' se već jednom." "Budge up there, move along.";

(2) the incentive and encouragement, e.g., "Hajde, postrojite se." "Now, form a line.";

(3) the incentive not expressed by a verb in the original text as a symptom of sarcastic stating, e.g., "Ma hajde, Hermiona, da li ti stvarno misliš da su svi profesori sveci ili nešto slično?" "Honestly, Hermione, you think all teachers are saints or something.";

(4) the expression of emotions and attitudes of the speaker, interjections and adverbs, for instance the expression 'well,' e.g., "Hajde, mrdni, hoću da pripaziš na slaninu." "Well, get a move on, I want you to look after the bacon."

(5) the imperative, which is in the original text formed just by using one verb, in other words, the construction ' hajde' plus a full-meaning verb in the imperative, for example 'hajde' + 'uzmi' (hajde + have/take) instead of one verb in imperative, 'have': "Hajde, uzmi čokoladnu žabicu, imam ih na tone..." "Have a Chocolate Frog, I've got loads..." 
(6) the so-called da-construction can be found as well ('hajde' + 'da' + verb in present tense, where the 'da' is a preposition) with the imperative meaning, e.g., "Hajde da oprostimo i zaboravimo." "Forgive and forget."

Our data also confirmed the occurrence of the terms 'hajdemo' and 'ajdemo' which are regularly formed as first-person plural forms of the verbs.

In the Serbian text, both versions of the word, 'hajde' and 'ajde,' appear. The remarkable point is that the form 'ajde' only appears when Hagrid speaks (but not exclusively, one occurrence proves form 'hajde' in Hagrid's speech). The specificity of Hagrid's character and his speech preceded.

The total number of occurrences of 'ajde' and 'hajde' or their variant is 42 in the Serbian text, with the word 'ajde' used five times, to maintain the sociolect, and the word form 'hajde' used 37 times, which proves it to be more widely used.

In the third text included in our research, the Croatian one, fewer examples of usage of the words 'ajde' and 'hajde' were found. In addition to the abovementioned meanings (come on, c'mon, and let's), the following contexts should be named:

(1) the expression of emotions and attitudes of the speaker, interjections and adverbs as 'well' or 'now,' e.g., “Hajde, požuri se da pripaziš na slaninu u tavi." "Well, get a move on, I want you to look after the bacon.”; "Hajde radije odlazite!" "Move along now.";

(2) the imperative originally formed just by one verb; thus, the construction 'hajde' plus a full-meaning verb in the imperative are used in the translation, for example, 'hajde' + 'požuri' (hajde + hurry up) instead of one verb in imperative 'hurry up': "Hajde požuri, dečko, nemamo cijeli dan vremena." "Hurry up, boy, we haven’t got all day.”; “Ali sad ti, Hermiona, možeš slobodno uzeti onog lovca, hajde, uzmi ga!" "Leaves you free to take the bishop, Hermione, go on.”; "Hajde sad, prolazite svi dalje!" "Move along, all of you!"

(3) the so-called da-construction that is found in the Serbian text was also found in the Croatian text, e.g., "Hajde, Potter, da još jednom pokušamo. Na koju ćeš stranu pogledati ako ti kažem da potražiš bezoar?" "Let's try again. Potter, where would you look if I told you to find me bezoar?"; "Hajde da se radije ogledaš s ovima." "Let's try you out with a few of these."

(4) on the other hand, in the Croatian text there can be found an accentuation of the expression of the imperative, e.g., "Hajde, hajde, Hermiona!" "Come on, Hermione." (the verb 'hajde' is used twice instead of once, unlike in the original text). 
The other contexts found in the Macedonian and Serbian translations-for example, the expression of the imperative of the verb of movement and the incentive not expressed by a verb in the original text as a symptom of a sarcastic statement-did not occur in the Croatian translation.

In both Croatian and Serbian texts, both of the words 'hajde' and 'ajde' appear. The form 'ajde' is used exclusively in Hagrid's speech. In contrast with the Serbian text, no other form occurs in Hagrid's speech.

The total number of occurrences of 'ajde' and 'hajde' or their variant is 29 in the Croatian text, with the word 'ajde' used five times, to maintain the sociolect, and the word form 'hajde' used 24 times.

\section{Conclusion and Discussion}

In summary, this paper argues that the three selected translations significantly differ in terms of using Balkanisms. We focused on contexts in which the word 'ajde' or 'hajde' occurs in translations from the English original to three target languages: Macedonian, Serbian, and Croatian. Based on our findings and analysis of the contexts, we can now answer the research questions.

To the first question "Does the width of the field of meaning of the words 'ajde' and 'hajde' differ on the one hand in dictionaries and on the other hand in written translated texts?" our answer is that according to our research the width of the field of meaning given in the selected dictionaries does not fully reflect the breadth of use of the two words. In this work, we offer the addition of contexts in which both words are used in individual languages. It is especially interesting to use these words to express irony and emotion.

To the second question "How much does the use of the words differ in the examined texts?" our answer is that because of using translations of a single text, we can assess how the contexts of usage differ in each language. It was important to use the text of a children's book, which was translated directly into the target languages. Most of the occurrences of the words 'ajde' and 'hajde' were discovered in the Macedonian text, fewer in the Serbian text, and the fewest in the Croatian text. From the given analysis of data, it is obvious that the context of the usage of the selected word differs widely. The widest usage has been found in the Macedonian text. The most impressive is the usage of 'ajde' or 'hajde' as a symptom of sarcasm, which was 
found in the Macedonian and Serbian texts. A finding which seems to be remarkable is that individual variants of the word can be used as a sign of specific speech or sociolect (Hagrid's and to a lesser extent Ron's speech). These variants differ significantly. Overall, our results demonstrate a strong effect of this Balkanism on the three selected languages, as well as differences and specificities in usage.

Although we have indicated that children's literature, and therefore its translations, often tend towards adaptations (Shavit 2006, 25) and that the language used is sometimes simplified (2002), we still believe that, as far as direct speech is concerned, children's literature and its translations are suitable for our research. Accurate and current language is used in the direct speech of children's books, also due to the requirement of fluency and the so-called "dual role" (Dollerup 2003, 99-100) and the role of "dual readership" (Jobe 1996, 513).

Since the focus of the present paper is the usage of the words 'ajde' and 'hajde' in translations, we have chosen only one source text and its translations to have the same type and same amount of text. It is worth noting that this may have limited our research to some extent. Thus, future research could continue to explore this topic, and it would be very helpful to comprehend the usage of the words 'ajde' and 'hajde' in its entirety. To ascertain how the word is used in real life, we plan to conduct sociolinguistic field research among language users. Furthermore, we believe future studies that would focus on the usages of lexical units in an interdisciplinary framework combining translation studies and comparative linguistics could provide more insights into the travel of words across languages and cultures. 


\section{References}

Anonymous. [2012?]. Хари Потер и каменот на мудроста. Вy Joanne Kathleen Rowling. Originally published as Harry Potter and the Philosopher's Stone (London: Bloomsbury, 2000).

Bogdanović, Nedeljko. 1987. Srpski dijalektološki zbornik [Serbian dialectological collection]. Vol. 33. Belgrade: Srpska akademija nauka i umetnosti.

Brumme, Jenny. 2012. Introduction to Translating Fictional Dialogue for Children and Young People, 7-13. Edited by Martin B. Fischer and Maria Wirf Naro. Berlin: Frank and Timme.

Bužarovska, Eleni. 2020. "The Contact Hypothesis Revised: DOM in the South Slavic Periphery." Journal of Language Contact 13 (1): 57-95. doi:10.1163/19552629bja10003.

Čapek, Jan. 2004. "Multilingvismus, globalizace a společný evropský referenční rámec." [Multilingualism, globalization, and the common European framework of reference.] In Motivace a multilingvismus ve výuce cizich jazyků: Sborník přispěvků z mezinárodní konference konané na Katedře cizích jazyků Univerzity Pardubice ve dnech 22-23 zárí 2003 [Motivation and multilingualism in foreign language teaching: Proceedings of contributions from the international conference held by the Department of Foreign Languages of the University of Pardubice on 22-23 September 2003], edited by Helena Jaklová. Pardubice: Univerzita Pardubice.

Crnković, Zlatko, trans. 2014. Harry Potter i kamen mudraca. By Joanne Kathleen Rowling. Zagreb: Algoritam. Originally published as Harry Potter and the Philosopher's Stone (London: Bloomsbury, 2000).

Dollerup, Cay. 2003. "Translation for Reading Aloud.” Traduction pour les enfants 48 (1-2): 81-103. doi:10.7202/006959ar.

Feral, Anne-Lise. 2006. “The Translator's 'Magic' Wand: Harry Potter's Journey from English into French.” Meta 51 (3): 459-481. doi:10.7202/013553ar.

Friedman, Victor A. 1994. "Turkisms in a Comparative Balkan Context." In Septième congrès international d'études du sud-est Européen (29 août-4 septembre 1994): Rapports [Seventh international congress for Southeastern European Studies (29 August-4 September 1994): Reports], 521-543. Athens: Greek National Committee for Southeast European Studies.

- 2011. "The Balkan Languages and Balkan Linguistics." Annual Review of Anthropology 40:275-291.

Gavurova, Miroslava. 2018. "Didaktika prekladu literatúry pre děti a mládež." [Didactics of translation of literature for children and youth.] In Didaktika prekladu a tlmočenia na 
Slovensku [Didactics of translation and interpreting in Slovakia], edited by Martin Djovčoš and Pavol Šveda, 70-101. Bratislava: Univerzita Komenského v Bratislavě.

Gramelová, Lucie. 2014. Albánština: lingvistický pohled [Albanian: a linguistic view]. Prague: Jaroslav Gramel.

Hauptová, Zoe. 1970. “Lexikální balkanismy v západoslovanských jazycích, zvláště v slovenštině." [Lexical balkanisms in west Slavic languages, especially in Slovak.] In Studia Balkanica Bohemoslovaca [Balkan Czech-Slovak Studies], edited by Richard Pražák and Ivan Dorovský, 281-290. Brno: Universita J. E. Purkyně. http://hdl.handle.net/11222.digilib/120697.

Hendrich, Josef et al. 1988. Didaktika cizich jazyků [Didactics of foreign languages]. Prague: SPN.

Hetemi, Atdhe. 2015. "Orientalism, Balkanism and the Western Viewpoint in the Context of Former Yugoslavia." ILIRIA International Review 5 (1): 311-335. doi:10.21113/iir.v5i1.22.

Inggs, Judith. 2003. "From Harry to Garri: Strategies for the Transfer of Culture and Ideology in Russian Translations of Two English Fantasy Stories." Meta 48 (1-2): 285-297. doi:10.7202/006975ar.

Jentsch, Nancy K. 2001. "Harry Potter Speaks in Tongues: Translating J. K. Rowling's Magical World.” Kentucky Philological Review 16:54-60.

— 2002. "Harry Potter and the Tower of Babel: Translating the Magic." In The Ivory Tower and Harry Potter: Perspectives on a Literary Phenomenon, edited by Lana Whited, 285-304. Columbia: University of Missouri Press.

Jobe, Ronald. 1996. "Translation." In International Companion Encyclopedia of Children's Literature, edited by Peter Hunt, 519-529. New York: Routledge.

Kahl, Thede. 2014. "Dynamics of the Common Balkan Lexemes: New Research Perspectives and Desiderata in the Field of Balkan Linguistics." In Die Welt der Slaven: Internationale Halbjahresschrift für Slavistik [The world of Slavs: International halfyearly publication for Slavic Studies], edited by Peter Rehder and Igor Smirnov, 59:310-331. Munich: Sagner.

Koneski, Kiril. 1999. Za makedonskiot glagol [On the Macedonian verb]. Skopje: Detska radost.

Kramer, Christina E., and Liljana Mitkovska. 2011. Macedonian: A Course for Beginning and Intermediate Students. Madison: University of Wisconsin Press.

Kurzová, Helena. 1995. “Gramatikalizační procesy v balkánských jazycích.” [Grammaticalization processes in Balkan languages.] In Studia Balkanica Bohemo- 
Slovaca IV [Balkan Czech-Slovak studies IV], edited by Ivan Dorovský, 76-88. Brno: Masarykova Univerzita.

Liškař, Čestmír. 1970. Transfer a interference ve vyučování cizím jazykům [Transfer and interference in foreign language teaching]. Brno: Krajský pedagogický ústav.

Madgearu, Alexander. 2008. The Wars of the Balkan Peninsula: Their Medieval Origins. Edited by Martin Gordon. Lanham: Scarecrow Press.

Novák, Pavel. 1979. "Nad dílem profesora Vladimíra Skaličky." [On the work of Professor Vladimír Skalička.] Slovo a slovesnost 40 (4): 345-349.

O'Connell, Eithne. 2006. "Translating for Children." In The Translation of Children's Literature: A Reader, edited by Gillian Lathey, 15-24. Clevedon: Multilingual Matters.

Piper, Predrag, and Milka Ivić. 2005. Sintaksa savremenoga srpskog jezika: Prosta rečenica [Syntax of the modern Serbian language: A simple sentence]. Belgrade: Institut za srpski jezik SANU.

Roganović, Draško, and Vesna Stamenković Roganović, trans. 2016. Hari Poter i kamen mudrosti. By Joanne Kathleen Rowling. Belgrade: Evro Giunti. Originally published as Harry Potter and the Philosopher's Stone (London: Bloomsbury, 2000).

Rowling, Joanne Kathleen. 2000. Harry Potter and the Philosopher's Stone. London: Bloomsbury.

Schwarz, Michal, and Václav Blažek. 2011. "Klasifikace a přehled turkických jazyků." [Classification and survey of Turkic languages.] Linguistica Brunensia 59 (1-2): 2559. http://hdl.handle.net/11222.digilib/115185.

Shavit, Zohar. 2006. “Translation of Children's Literature.” In The Translation of Children's Literature: A Reader, edited by Gillian Lathey, 25-40. Clevedon: Multilingual Matters.

Skok, Petar. 1971. Etimologijski rjecnik hrvatskoga ili srpskoga jezika [Etymological dictionary of the Croatian or Serbian language]. Zagreb: Jugoslavenska Akademija Znanosti i Umjetnosti.

Stachowski, Marek. 2019. "Slavic Languages in Contact, 2: Are There Ottoman Turkish Loanwords in the Balkan Slavic Languages?" Studia Linguistica Universitatis Iagellonicae Cracoviensis 136 (2): 99-105. doi:10.4467/20834624SL.19.009.10604.

Stevanović, Mihailo. 1970. Savremeni srpskohrvatski jezik [Contemporary Serbo-Croatian language]. Vol. 2. Belgrade: Naučna knjiga.

Tabbert, Reinbert. 2002. "Approaches to the Translation of Children's Literature: A Review of Critical Studies since 1960.” Target 14 (2): 303-351. doi:10.1075/target.14.2.06tab. 
transLogos 2020 Vol 3 Issue 2

trans Logos

Dufková, Kristýna, pp. 62-82

A Translation Studies Journal

'Ajde' and 'Hajde': Contexts of the Use of Balkanisms

in Translations into South Slavic Languages

(C) Diye Global Communications diye.com.tr|diye@diye.com.tr

Todorova, Maria. 2004. Imagining the Balkans. Oxford: Oxford University Press. 